\title{
Preprocessing To Be Considered For MR and CT Images Containing Tumors
}

\author{
Sonali Patil ${ }^{1}$, Dr. V. R. Udupi ${ }^{2}$ \\ ${ }^{I}$ (Ph.D. Scholar, Shivaji University, Kolhapur, Associate Professor, Department of IT, KJSCE, Mumbai, India) \\ ${ }_{2}^{2}$ (Professor, Department Of Electronics and Communication Engineering, GIT, Belgaum- 590 008, India)
}

\begin{abstract}
Image pre-processing is an important and challenging factor in the computer-aided diagnostic systems. In medical image processing and especially in tumor segmentation task it is very important to preprocess the image so that segmentation and feature extraction algorithms work correctly. Proper detection and segmentation of the tumor leads to exact extraction of features and classification of those tumors. The accurate tumor segmentation is possible if image is pre-processed as per image size and quality. This paper describes the pre-processing method consisting of two phases. In the first phase we remove the film artifact by using median filter. In the second phase we introduce an algorithm that uses morphological operations to remove unwanted skull/ribcage portion. This reduces the false positive results in the later stages of processing in the computeraided diagnostic systems .Both algorithms are applied on MR/CT images of Brain having tumors and, CT images of Thorax and Abdomen having tumors. The second algorithm has an effect of skull portion removal in Brain images and, effect of ribcage portion removal in CT images of Thorax and Abdomen. The results are superior to those of tracking algorithms considering the retention of regions of interests and the film artifacts removal. This preprocessing will also reduce the over segmentation problem in further processing still retaining the tumors.
\end{abstract}

Keywords: Pre-processing, Medical Imaging, Tumors, Median Filter, Morphological Operators

\section{Introduction}

Medical imaging plays an important role in patient diagnosis. To examine the disease of patient imaging technology is used. MRI and CT provide good contrast between the different soft tissues of the brain. Medical imaging is an essential component for application in the clinical track of events including clinical diagnostic settings, planning, consummation, and evaluation of surgical and radiotherapeutical procedures. The computer-aided diagnosis system (CAD) is helpful for clinical diagnosis and treatment. MRI and CT are vital diagnostic imaging techniques. The x-ray computerized axial tomography (CT) provides a 2D map of the line attenuation coefficient mirroring morphological details of the organs under study. X-ray CT is a high spatial resolution and wide dynamic range imaging modality, so, even small density abnormalities can be detected [1].A CT image can be obtained within one breath hold which makes CT the modality of choice for imaging the thoracic cage. Magnetic Resonance Imaging (MRI) technique utilizes strong magnetic fields and non-ionizing radiation in the radio frequency range, and according to current medical knowledge, is harmless to patients. Another advantage of MRI is that soft tissue contrast is much better than with X-rays leading to higher-quality images, especially in brain and spinal cord scans.

\subsection{Need for pre-processing}

The pre-processing is required for MR/CT images as:

- Marks or labels present (Film artifacts) can interfere in the post-processing of these images.

- Images are to be made more suitable for further processing in CAD systems.

- The image quality needs to be enhanced

- Noise in the image needs to be removed.

Here an attempt has been made to identify the suitable algorithms for pre processing the MR/CT images having tumors. The objective of this work is to:

a. Remove the film artifacts and skull portions from the Brain MR/CT images and

b. Remove the skull/ribcage portions from the CT images of Thorax/Abdomen.

\subsection{Related work}

The MRI/CT image consists of film artifact or labels such as patient name, age and marks. In a wide variety of image processing applications, it is necessary to smooth an image while preserving its edges. The gray levels often overlap that makes any post-processing task such as segmentation [4-7], feature extraction and 
labeling more difficult. Filtering is perhaps the most fundamental operation in many biomedical image processing applications, where it reduces the noise level and improves the quality of the image.

Film artifacts are removed using tracking algorithm. As mentioned in [2] we can use tracking algorithm to remove artifacts in Brain MR images. But the tracking algorithm uses highest threshold for gray scale image which is 255 . This will remove artifacts which have a gray value of 255 only leaving all other artifacts on the image. This algorithm also removes data from ROI. The tracking algorithm used for removal of skull portions in the brain MR image proposed in [2] uses threshold [200 -255] to remove skull portions. This will additionally remove required data form ROI located adjacent to skull. These results are shown in Fig1. The limitations of these algorithms are clearly seen from the result.

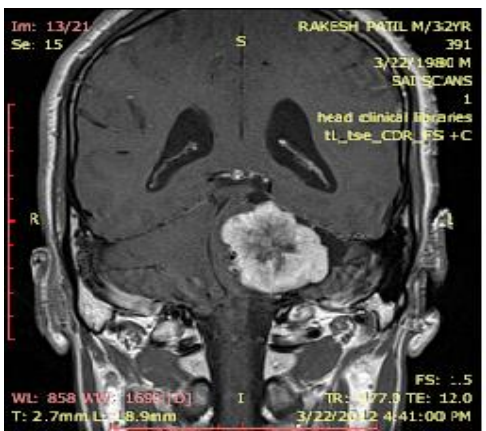

(a) Original image

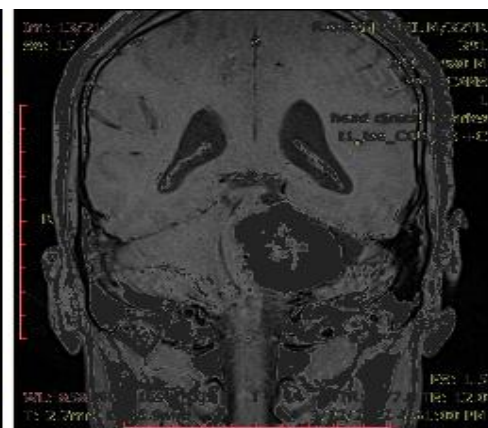

(b) Output of tracking algorithms

Fig 1.

\section{Proposed Methods}

In this paper two methods are proposed. One method is for removal film artifacts and, other for removal of skull portions from brain image and ribcage portions from the Thorax and Abdomen images.

\subsection{Removal of film artifacts from MRI/ CT images by median filtering}

The main idea of the median filter [3] is to run through the signal entry by entry, replacing each entry with the median of neighboring entries. The pattern of neighbors is called the "window", which slides, entry by entry, over the entire signal. For 1D signal, the most obvious window is just the first few preceding and following entries, whereas for 2D (or higher-dimensional) signals such as images, more complex window patterns are possible (such as "box" or "cross" patterns). Note that if the window has an odd number of entries, then the median is simple to define: it is just the middle value after all the entries in the window are sorted numerically. For an even number of entries, there is more than one possible median.

\subsubsection{Window possibilities:}

Square Mask
x x x
X x x
x x x

Plus Mask
x
x x x
X

X Mask
$x \quad x$
$x$
$x \quad x$

Here we apply simple box window to remove artifact from input image. This method will remove artifacts from image and will also retain the data from ROI (in this case tumor). This method is beneficial as it keeps data as well as remove noise from input image. This algorithm is efficient as compared to tracking algorithm and provides output images that are suitable for further processing.

\subsection{Removal of Skull portions from Brain MRI/CT images using Morphological Erosion}

Many times it is required to remove skull portions from the brain images. The skull portion is bright and this may hamper the segmentation process. Here we propose a morphological operator base algorithm to remove the skull portions. The same algorithm is applied on Abdomen and Thorax CT scans for removal of ribcage portions.

\subsubsection{Morphology:-}

Mathematical morphology is based on set theory. It is a way of treating images without altering the shape of the objects in the images [2]. The shapes of objects in a binary image are represented by object membership sets. This theory can be extended to gray scale images. Morphological operations can simplify image data, preserving the objects' essential shape characteristics, and can eliminate irrelevant objects. 
Preprocessing To Be Considered For MR and CT Images Containing Tumors

Mathematical morphology has two fundamental operations, dilation, which fills holes and smoothens the contour lines, and erosion, which removes small objects and disconnects objects connected by a small bridge [2] . Such operations are defined in terms of a structuring element, a small window that scans the image and alters the pixels in function of its window content. The choice of the proper structuring element is important.

The main advantage of a morphological filter is the ability to preserve the shape of large enough objects, unlike a Gaussian filter which blurs the image. This makes morphological operations appropriate for image pre-processing.

\subsubsection{Proposed algorithm}

Step1.Load the image

Step2.Apply the median filtering algorithm to remove the artifacts

Step3. Use square shaped structuring element

Step4. Apply erosion using Structuring Element three times but every time change the size of structuring element

1 st time we keep size $4 * 4$

2nd time we keep size $3 * 3$

3rd time we keep size $2 * 2$

Step5. After applying step4 we get the image without skull

\section{Results}

The algorithms were applied on 30 different MRI/CT scans of Brain, Thorax and Abdomen. The results of pre-processing by both the algorithms used are shown in fig. 2 - fig.5. The results show that the artifacts from all the images were satisfactorily removed by the first algorithm. In Brain MR Images the skull portions are efficiently removed. The second algorithm when applied on CT images of Thorax and Abdomen significantly reduces the effect of ribcage. The tumors are retained in all the images.

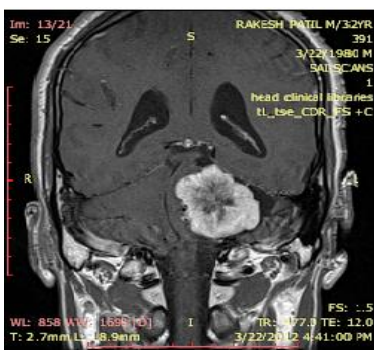

a. MR Brain image

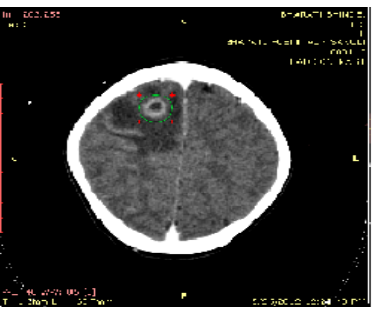

a. CT Brain image

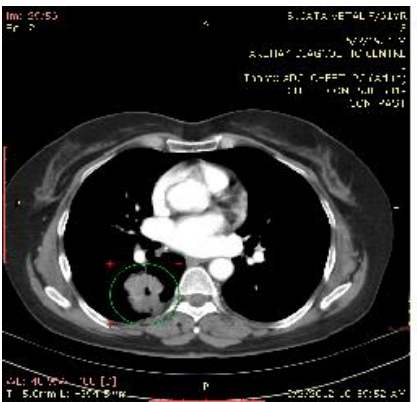

a. Thorax CT showing Lung

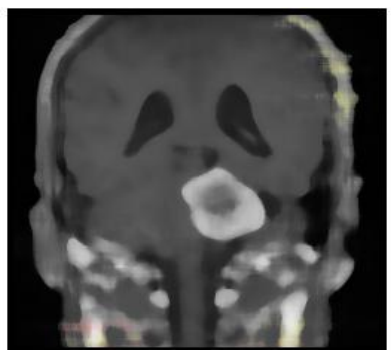

b. Output of first algorithm Fig.2

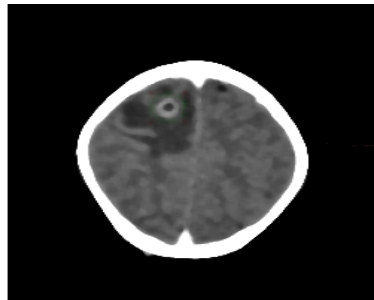

b. Output of first algorithm Fig. 3

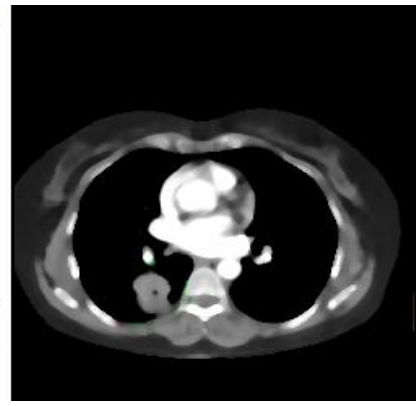

b. Output of first algorithm Fig.4

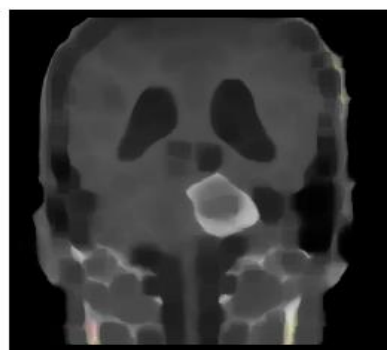

c. Output of second algorithm

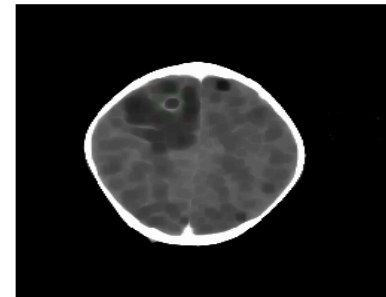

c. Output of second algorithm

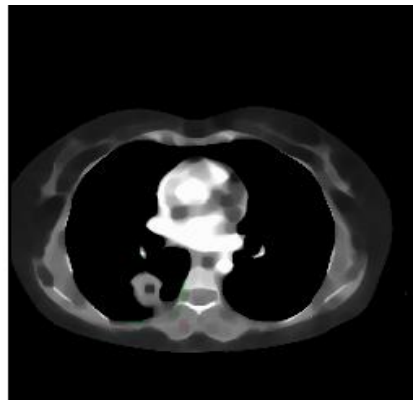

c. Output of second algorithm 


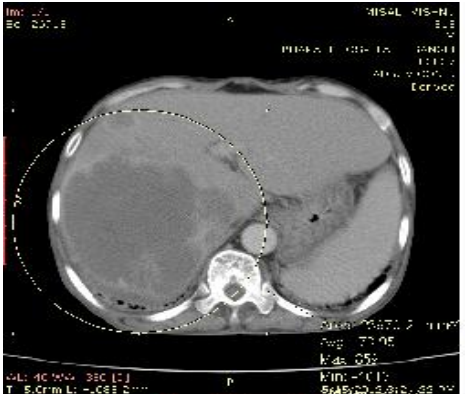

a. Abdomen CT showing Liver

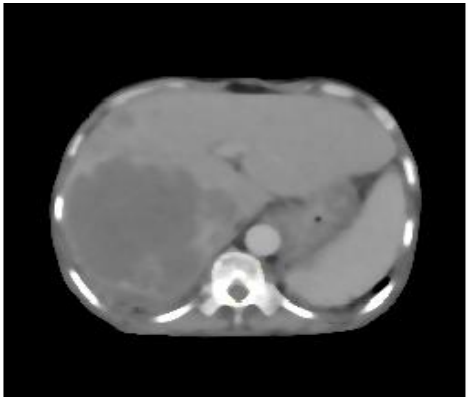

b. output of first algorithm Fig.5

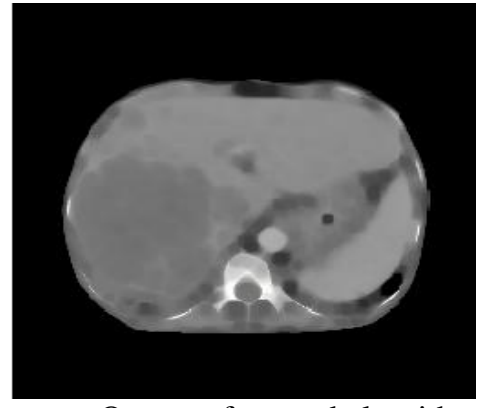

c. Output of second algorithm

\section{IV.}

\section{CONCLUSION}

We applied both the algorithms on different CT and MRI images of Brain, Thorax and Abdomen. The film artifacts and unwanted portions of Brain MR Images were successfully removed. The results were compared with that of tracking algorithms and were proved to be significantly better considering removal of film artifacts and retention of ROIs (i.e. Tumors). The film artifacts from all the CT images were successfully removed retaining the tumors. The salt and pepper noise additionally gets removed from all the images along with the film artifacts. In case of CT Brain images as the skull portion is thicker, the skull portion is thinned and not completely removed. In case of CT images of Thorax and Abdomen the effect of ribcage portion is significantly minimized. The images pre-processed in this manner are such that they remove the unwanted portions from the images thereby significantly reducing the chances of over-segmentation in the process of segmentation of these images.

\section{Acknowledgements}

Authors sincerely would like to thank the management of the Siddhivinayak Cancer Hospital, Bharati Hospital and Akshay Diagnostic Centre, for providing the MR and CT images. We would also like to thank Dr. B. M. Patil and Dr. Bharat Mudalgi, for their valuable inputs on aspects of Medical Imaging.

\section{REFERENCES}

[1] Sigurd Angenent, Eric Pichon, Allen Tannenbaum, Mathematical Methods in Medical Image Processing, BULLETIN (New Series)OFTHEAMERICANMATHEMATICALSOCIETY,00(0),Pages000-000. www.math.wisc.edu/ angenent/preprints/medicalBAMS.pdf)

[2] Jaya , K.Thanushkodi , M.Karnan, Tracking Algorithm for De-Noising of MR Brain Images, International Journal of Computer Science and Network Security, 9(11), November 2009, 262-267

[3] Rafael C. Gonzalez, Richard E. Woods, Digital Image Processing (Prentice Hall, Aug 21, 2007)

[4] Pan Lin ,Yong Yang, Chong-Xun Zheng, Jian-Wen Gu,.An Efficient Automatic Framework for Segmentation of MRI Brain Image, Proceedings of the Fourth International Conference on Computer and Information Technology(CIT'04),IEEE,2004.

[5] K.Selvanayaki, Dr. M. Karnan, $C A D$ System for Automatic Detection of Brain Tumor through Magnetic Resonance Image-A Review, International Journal of Engineering Science and Technology, 2(10), 2010, 5890-5901.

[6] Harman Kataria, Alka Jindal, Brain MRI: Region based Segmentation Techniques, IJCST, 3(1), Jan. - March 2012,527-529.

[7] Wang Rongfu, PET/CT Tumour Diagonsis, Peking University Medical Press, 1st ed., Beijing, 2007, pp. 3-13.(in Chinese)

[8] Csaba Barcsák, Péter Mileff, Preprocessing and Real Time Visualization of CT and MRI Data, microCAD 2011, 25th International Scientific Conference, University of Miskolc Hungary, pp 7-12

[9] José Silvestre Silva, Augusto Silva and Beatriz Sousa Santos, Lung Segmentation Methods in X-ray CT Images, SIARP2000, 5TH IBEROAMERICAN SYMPOSIUM ON PATTERN RECOGNITION, 583-598. 